\title{
Tratamento cirúrgico da endocardite infecciosa
}

\author{
Gustavo Roberto HOPPEN*, Iselso Paulo SARTORI*, Luís Sérgio FRAGOMENI*
}

RBCCV 44205-275

\begin{abstract}
HOPPEN, G.R.; SARTORI, I.P.; FRAGOMENI, L. S. - Tratamento cirúrgico da endocardite infecciosa. Rev. Bras. Cir. Cardiovasc., 10 (4): 175-179, 1995.

RESUMO: RAvaliaram-se as indicações, as alterações e os resultados cirúrgicos de 28 pacientes, operados por endocardite infecciosa (EI) no período de 1983 a 1994 . O diagnóstico clínico foi confirmado pela ecocardiografia e pela cineangiocardiografia. Dos pacientes, $65 \%$ eram do sexo masculino e $25 \%$ do feminino, com média de idades de 35,21 (14 a 67) anos. Noventa e três por cento dos pacientes estavam em classe funcional III e IV (NYHA). O tempo decorrido entre o diagnóstico e a cirurgia foi em média de quatro semanas e meia (1 a 363 dias). A hemocultura foi positiva em $25 \%$ dos casos e os germes mais freqüentemente encontrados foram estreptococos e estafilococos. A valva aórtica foi a mais acometida e as vegetaçöes foram as lesões mais comumente encontradas. O tratamento cirúrgico utilizado foi a plastia valvar em 2 casos e a substituição valvar por prótese em 26. Complicações ocorreram em $39,28 \%$ dos casos, com $5(18 \%)$ mortes. Conclui-se que, embora rara, a El é doença grave e, se não prevenida com adequada antibiótico-profilaxia ou tratada em tempo hábil, o paciente terá conseqũências graves, podendo, até mesmo, falecer.
\end{abstract}

DESCRITORES: Endocardite infecciosa, cirurgia.

\section{INTRODUÇÃO}

A endocardite infecciosa (EI) pode ser definida como uma doença decorrente da infecção do endotélio das valvas e câmaras cardiacas 10 .

Estima-se uma incidência anual de 3,8 casos em cada 100 mil pessoas e uma prevalência de 25 casos por 1 milhão de habitantes ${ }^{16}$.

Procurou-se avaliar, neste trabalho, as indicações mais freqüentes para o tratamento cirúrgico da $\mathrm{EI}$, as lesões encontradas no transoperatório e os resultados a médio prazo deste tratamento cirúrgico em um grupo de 28 pacientes, no Serviço de Cirurgia Cardiotorácica do Hospital Universitário São Vicente de Paulo (HSVP), em Passo Fundo, RS.

\section{CASUÍSTICA E MÉTODOS}

Foram revisados os prontuários de 28 pacientes submetidos a cirurgia cardíaca em razão de El, no período de 1983 a 1994, no Serviço de Cirurgia Cardiotorácica do HSVP. Para cada paciente, foi preenchida uma ficha com os seguintes dados: idade, sexo, classe funcional (NYHA) pré-operatória, uso ou não de respirador no pré-operatório, presença de embolia periférica, embolia cerebral, sepse ou choque, presença ou não de infecção no momento da cirurgia, tempo decorrido entre o diagnóstico e - tratamento cirúrgico, métodos utilizados para o diagnóstico, resultado da hemocultura, tempo de antibioticoterapia prévia, cirurgia cardíaca prévia, valva acometida, indicação cirúrgica, lesão encon-

Trabalho realizado no Serviço de Cirurgia Cardiotorácica do Hospital São Vicente de Paulo. Departamento de Cirurgia da Faculdade de Medicina da Universidade de Passo Fundo, RS, Brasil.

Recebido para publicaçăo em novembro de 1995.

Endereço para correspondência: Gustavo Roberto Hoppen. Rua Uruguai, 1555. CEP 99010-112 Passo Fundo, RS, Brasil. 
HOPPEN, G.R.; SARTORI, I. P.; FRAGOMENI, L. S. - Tratamento cirúrgico da endocardite infecciosa. Rev. Bras. Cir. Cardiovasc., 10 (4): 175-179, 1995.

trada no transoperatório, procedimento cirúrgico realizado e complicações pós-operatórias.

Vinte e um (75\%) pacientes eram do sexo masculino e $7(25 \%)$ do feminino, com média de idades de 35,21 anos (14 a 67 anos). Em relação à classe funcional no pré-operatório, $19(67,8 \%)$ pacientes estavam em classe IV, 7 (25\%) em classe III e apenas $2(7,1 \%)$ em classe II, conforme demonstrado na Tabela 1. Nenhum dos pacientes necessitou de suporte ventilatório no pré-operatório. Dois pacientes tinham sinais clínicos de embolia cerebral, tendo um deles apresentado embolia periférica associada a quadro séptico. Em $6(21,4 \%)$ casos, havia septicemia no pré-operatório.

TABELA 1

CLASSE FUNCIONAL NO PRÉ-OPERATÓRIO

\begin{tabular}{ccc}
\hline Classe & Número & $\%$ \\
\hline II & 2 & 7,1 \\
III & 7 & 25,0 \\
IV & 19 & 67,8 \\
\hline
\end{tabular}

O tempo decorrido entre o diagnóstico e a cirurgia variou de um dia até um ano, com média de 32,54 dias.

Em todos os pacientes, o diagnóstico foi sugerido pela clínica apresentada e, em $17(60,71 \%)$, foi confirmado pelo estudo ecocardiográfico isolado. Foi utilizada a cineangiocardiografia isolada em apenas 1 e, em $10(37,5 \%)$ pacientes, foi necessária a associação deste estudo com a ecocardiografia. A hemocultura foi realizada em todos os pacientes, sendo positiva em $25 \%$. Das culturas positivas, o estreptococo foi o germe mais freqüente $(57 \%)$, seguido pelo estafilococo $(28,57 \%)$. Identificou-se o germe Klebsiella em apenas uma cultura.

Sete $(25 \%)$ pacientes tinham diagnóstico de lesão cardíaca prévia, a maioria por insuficiência aórtica. As demais lesões estão demonstradas na Tabela 2. Destes pacientes, 4 haviam sido submetidos a cirurgia cardíaca prévia.

TABELA 2

LESÃO CARDÍACA PRÉVIA À CIRURGIA

\begin{tabular}{lc}
\hline Tipo & Casos \\
\hline Estenose aórtica & 1 \\
Insuficiência aórtica & 3 \\
Prolapso de valva mitral & 1 \\
Coartação de aorta & 1 \\
Comunicação interventricular & 1 \\
\hline Total & $7 / 28$ \\
\hline
\end{tabular}

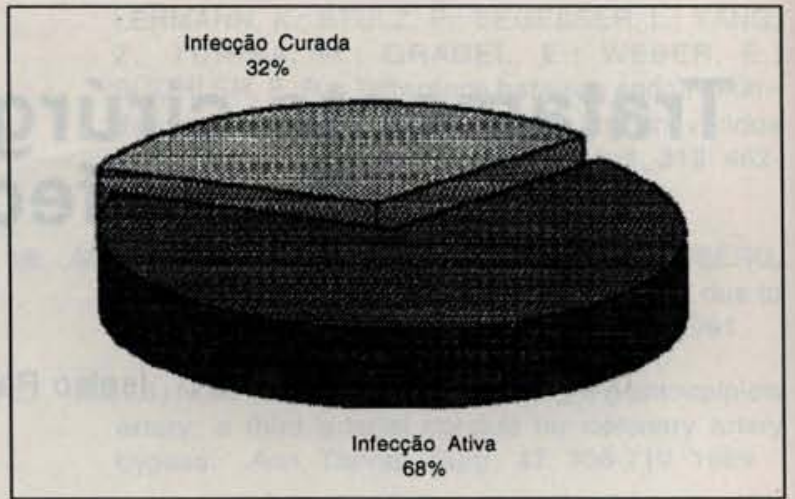

Fig. 1 - Porcentagem de infecção no pré-operatório.

Doze $(42,8 \%)$ pacientes foram operados por insuficiência cardíaca descompensada e outros 12 por infecção não controlada com antibioticoterapia. Três $(10,7 \%)$ pacientes foram submetidos a cirurgia devido à presença de El em prótese valvar cardiaca, sendo todas biológicas. Indicou-se a cirurgia em 1 caso, pela associação de El com bloqueio átrioventricular de $3^{\circ}$ grau.

A infecção foi considerada ativa em $67,85 \%$ dos casos, conforme demonstrado na Figura 1.

O acometimento isolado da valva aórtica foi o responsável pela maioria dos casos, seguido pelo envolvimento isolado da valva mitral, como demonstrado no Gráfico 1

GRÁFICO 1

Valvas cardiacas acometidas.

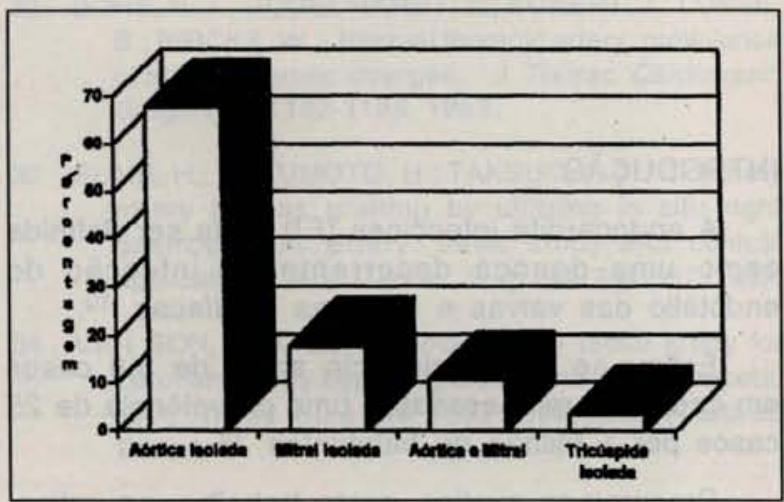

\section{RESULTADOS}

No transoperatório, foi possivel observar a existência, em quase todos os pacientes, de mais de um tipo de lesão (Tabela 3).

A Figura 2 demonstra os métodos de correção cirúrgica neste grupo de pacientes.

O tempo de pinçamento aórtico variou de 25 a 120 minutos (média de 69,8 ). O tempo médio de permanência em CTI foi de 4,28 dias. 
HOPPEN, G.R.; SARTORI, I. P.; FRAGOMENI, L. S. - Tratamento cirúrgico da endocardite infecciosa. Rev. Bras. Cir. Cardiovasc., 10 (4): 175-179, 1995.

TABELA 3

LESÕES ENCONTRADAS NA OPERAÇÃO

\begin{tabular}{lcc}
\hline Lesões Isoladas & Pacientes & $\%$ \\
\hline Vegetações & 22 & 78,57 \\
Abscesso perianular & 6 & 21,42 \\
Rotura de cordas & 1 & 3,57 \\
Disfunção de prótese & 1 & 3,57 \\
Reabsorção de válvulas & 1 & 3,57 \\
Fistula AE/AO & 1 & 3,57 \\
\hline
\end{tabular}

Ocorreram complicações em $11(39,28 \%)$ pacientes, sendo a pneumonia com manifestações clinicas e radiológicas o achado mais comum. Quatro pacientes necessitaram de mais de uma intervenção cirúrgica, 3 por deiscência da prótese e 1 por insuficiência mitral pós-plastia. Houve cinco óbitos, tendo sido operados 4 pacientes na presença de infecção ativa e insuficiência cardíaca classe IV (Tabela 4).

TABELA 4

COMPLICAÇŌES NO PERIODO DE PÓS-OPERATÓRIO

\begin{tabular}{ll}
\hline Complicações & Casos \\
\hline Óbitos: choque séptico (4) & \\
$\quad$ falência miocárdica 10 & p.o. (1) \\
Pneumonia & 5 \\
Embolia periférica & 3 \\
Fístula perivalvar & 1 \\
Fistula átrio direito-aorta & 3 \\
Falência de plastia mitral & 1 \\
\hline
\end{tabular}

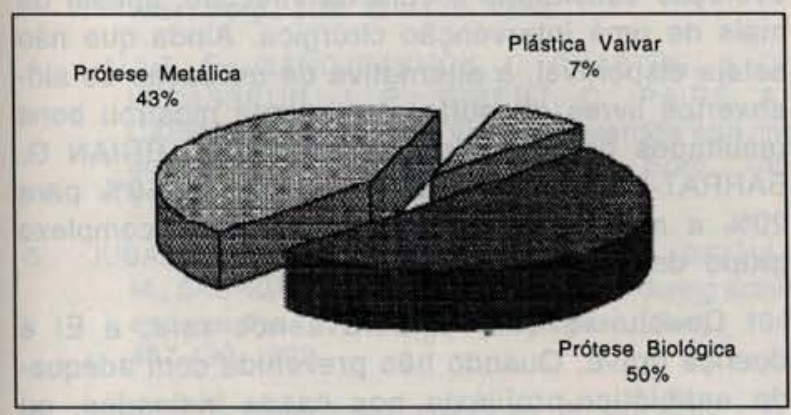

Fig. 2 - Métodos de correção cirúrgica.

\section{COMENTÁRIOS}

Embora rara, a El é uma doença grave, apresentando taxas de mortalidade que variam de $40 \%$, em pacientes com infecção em valvas nativas, até cerca de $80 \%$, quando a infecção se estabelece em uma prótese valvular ${ }^{6}$. Três fatores hemodinâmicos predispõem os pacientes ao desenvolvimento de $\mathrm{El}$ : 0 jato sangüíneo com alta velocidade, o fluxo entre uma câmara de alta para uma de baixa pressão e um orifício relativamente estreito separando duas câmaras 8 .

Os sintomas geralmente aparecem duas semanas após a bacteremia. As manifestações clínicas desencadeadas são decorrentes do processo infeccioso instalado (mal-estar, febre, anorexia, emagrecimento), das alterações da anatomia cardíaca (disfunção valvar ou agravamento de disfunção preexistente), de embolias resultantes da liberação de fragmentos de vegetações e do estímulo antigênico prolongado 10 .

As indicações para o tratamento cirúrgico da $\mathrm{El}$, descritas na literatura ${ }^{10}$, são semelhantes às desta série e podem ser agrupadas da seguinte forma: grave insuficiência cardiaca e progressiva, refratária ao tratamento clínico; infecção não controlada com tratamento antimicrobiano; infecção complicada em prótese valvular cardíaca e embolias arteriais sistêmicas. Quanto à classe funcional, assim como demonstrado por outros autores $2,4,5,17$, observouse que a maioria dos pacientes encontrava-se nas classes III e IV da NYHA. Apenas $2(7,1 \%)$ pacientes apresentaram manifestações embólicas, valor este inferior ao apresentado pela literatura, que está entre $12 \%$ e $15 \%$. Septicemia esteve presente em $21,4 \%$ dos casos, resultado inferior aos de McGIFFIN et alii ${ }^{12}(37,5 \%)$.

O estudo ecocardiográfico transtorácico demonstrou as lesões causadas pela El em cerca de $78 \%$ dos pacientes estudados. Segundo KARZENIOWSKI et alii ${ }^{8}$ e MANSUR ${ }^{9}$, esse exame possui uma sensibilidade, na doença em questão, de $60 \%$ a $80 \%$. O achado de vegetações no ecocardiograma é complementar aos dados clínicos e à hemocultura ${ }^{10}$; no entanto, deve-se salientar que a ausência de vegetações não exclui o diagnóstico de EI ${ }^{10}$.

A hemocultura é considerada o exame complementar mais importante para o diagnóstico da El; quando usada na ausência de terapia antimicrobiana prévia, é positiva em mais de $95 \%$ dos pacientes 6,8 . Pode-se justificar o achado de apenas $25 \%$ de positividade nas hemoculturas, pelo fato de os pacientes terem sido manipulados com antibióticos nos hospitais de origem. Também deve-se referir à possibilidade de infecção por Haemophilus parainfluenzae, microorganismo delicado e que geralmente não é identificado na hemocultura ${ }^{6}$.

Em relação ao agente etiológico, o estreptococo predominou, sendo seguido pelo estafilococo. Existe uma grande variação na experiência de vários autores: alguns deles ${ }^{2,3}$ citam o estreptococo como 0 agente mais freqüente, e outros $5,8,12,13,15$, o estafilococo.

Apesar de ter sido identificado algum tipo de 
HOPPEN, G.R.; SARTORI, I. P.; FRAGOMENI, L. S. - Tratamento cirúrgico da endocardite infecciosa. Rev. Bras. Cir. Cardiovasc., $10(4): 175-179,1995$.

lesão cardíaca prévia ao quadro de El apenas em um quarto dos pacientes, sabe-se que ela é muito mais freqüente neste grupo de pacientes ${ }^{10}$. Este fato justifica a antibiótico-prolifaxia em pacientes com lesões cardíacas predisponentes que se submetem a procedimentos que, sabidamente, causam bacteremia (procedimentos invasivos em cavidade oral, nariz, orofaringe, trato gastrintestinal e geniturinário) 6 .

Dentre as indicações para o procedimento cirúrgico, a insuficiência cardiaca e a infecção não controlada com antibiótico adequado são as mais comuns $4,8,17$. Na nossa casuística, estas foram responsáveis por cerca de $90 \%$ das indicações cirúrgicas. A indicação menos freqũente foi a associação de EI com BAV de terceiro grau, encontrada em porcentagem muito menor que a citada por KORSENIOWSKI et alii ${ }^{8}$. Apesar de 10,7\% dos pacientes terem sido submetidos à cirurgia pela presença de El em prótese valvular, sabe-se que o risco atual de desenvolvimento desta doença nestes casos varia de $2 \%$ a $5,7 \% 1,14$, sendo esta uma indicação inequívoca para o tratamento cirúrgico da EI.

O acometimento isolado da valva aórtica foi responsável pela maioria dos casos, como demonstrado por WATANABE et alii ${ }^{17}$. O comprometimento combinado das valvas aórtica e mitral foi responsável por cerca de $10 \%$ dos casos, valor compatível aos já citados 5,17 .

HAYDOCK et alii ${ }^{2}$ relataram que as vegetações estão presentes na grande maioria dos pacientes. Neste estudo, encontrou-se cerca de $78,57 \%$ dos pacientes com vegetações. Como demonstraram JUBAIR et alii ${ }^{5}$, vê-se que a segunda lesão mais prevalente é o abscesso.

$\mathrm{Na}$ maioria dos casos, o procedimento cirúrgico indicado é a substituição valvar por prótese biológica ou metálica, sendo possível a realização de plástica valvar apenas em casos selecionados 10 . Considerando essas limitações, 2 pacientes deste grupo apresentavam condições para serem submetidos à plástica valvar; a maioria, no entanto, foi submetida à substituição valvar por prótese biológica ${ }^{11}$.

Dos pacientes estudados, a maioria foi operada com infecção ativa. Observou-se que, apesar da ocorrência de complicações em 11 pacientes, grande parte ( 9 casos) ocorreu em pacientes com infecção ativa na época da cirurgia. Quatro pacientes deste grupo faleceram. Com base nestes dados, pode-se inferir que a infecção ativa na época da cirurgia pode predispor a um maior número de complicações no pós-operatório, apesar de, em muitas ocasiōes, ser necessário realizar a operação nestas condiçōes. É, pois, necessário insistir que a opção cirúrgica não seja considerada apenas na falta de controle da sepse e falência ventricular grave. Assim como demonstrado por HAYDOCK et alii $^{2}$, a média de tempo decorrido entre o diagnóstico e a cirurgia foi de, aproximadamente, quatro semanas e meia. Extensa destruição tecidual valvar e de estruturas anexas, especialmente quando a valva aórtica é a acometida, é causa comum de desgarramento da prótese, causando fístulas e vazamento perivalvar. Foi esta a principal causa de reintervenção em 4 pacientes. Um deles com extensa destruição valvar aórtica e fístula aorta/átrio direito, necessitou de três intervençōes cirúrgicas até o controle da infecção e quadro clínico. Extenso envolvimento das estruturas intracardíacas, principalmente abscessos de septo interventricular, fístulas, envolvimento mitro-aórtico e bloqueio atrioventricular completo são causas de elevada mortalidade neste subgrupo de pacientes. Julga-se, nestas situações, ser imperioso que todo material necrótico seja removido; a utilização de enxertos de pericárdio pode ser uma solução satisfatória na reconstrução anatômica. Dois dos pacientes tiveram extensa ressecção e reparos desta forma, com evolução satisfatória e cura da infecção, apesar de mais de uma intervenção cirúrgica. Ainda que não esteja disponível, a alternativa de utilização de aloenxertos livres de aorta ascendente mostrou bons resultados na experiência do professor BRIAN G. BARRAT-BOYES ${ }^{1}$, quando reduziu de $50 \%$ para $20 \%$ a mortalidade em 6 meses nesse complexo grupo de pacientes.

Conclui-se que, embora sendo rara, a EI é doença grave. Quando não prevenida com adequada antibiótico-profilaxia nos casos indicados, ou tratada adequadamente, pode ser acompanhada de alta morbidade e mortalidade. 
HOPPEN, G.R.; SARTORI, I. P.; FRAGOMENI, L. S. - Tratamento cirúrgico da endocardite infecciosa. Rev. Bras. Cir. Cardiovasc., $10(4): 175-179,1995$.

RBCCV 44205-275

HOPPEN, G. R.; SARTORI, I. P.; FRAGOMENI, L. S. - Surgical treatment of infective endocarditis. Rev. Bras. Cir. Cardiovasc., $10(4):$ 175-179, 1995.

ABSTRACT: The indication, surgical pathology and the results of surgical treatment of 28 patients operated on for infectious endocarditis between 1983 and 1994 are here evaluated. The clinical diagnosis was confirmed by echocardiography and cineangiography. Seventy-five percent of the patients were male and $25 \%$ female, with the mean age of 35.2 (14 to 67 ) years old. Ninety three percent were in functional class III or IV ( $N$ Y H A). Elapsed time between diagnosis and surgery was around 4 weeks (1-363 days). A positive hemoculture was present in $25 \%$ of the cases and the commonest bacteria found were streptococcus and staphilococcus. The aortic valve was the one usualy affected and vegetations was the usual finding. Surgical treatment consisted of valvuloplasty in 2 cases and valve replacement in 26 . Complications were present in $39.28 \%$ of the cases, which resulted in the death of five of them $(18 \%)$. We conclude that, although rare, the infectious endocarditis is a serious disease; if not prevented with adequate antibiotic prophilaxis or treated in due time, serious complications or even death might occur.

DESCRIPTORS: Endocarditis, infeccious, surgery

\section{REFERÊNCIAS BIBLIOGRÁFICAS}

1 CALDERWOOD, S. B.; SWINSKI, L. A.; KARCHMER, A. W.; WATERNOUX, C. M.; BUCKLEG, M. J. Prosthetic valve endocarditis: analysis of factors affecting outcome of therapy. J. Thorac. Cardiovasc. Surg., 92: 776-783, 1986.

2 HAYDOCK, D.; BARRAT-BOYES, B.; MACEDO, T.; KIRKLIN, J. W.; BORST, H. G. - Aortic valve replacement for active infectious endocarditis in 108 patients. J. Thorac. Cardiovasc. Surg., 103: 130139, 1992.

3 HENDREN, W. G.; MORRIS, A. S.; ROSENKRANZ, E. R.; LYTLE, B. W.; TAYLOR, P. C.; STEWART, W. J.; LOOP, F. D.; COSGRAVE, D. M. - Mitral valve repair for bacterial endocarditis, J. Thorac. Cardiovasc. Surg., 103: 124-129, 1992.

4 JAULT, F.; GANDJBAKHCK, I.; CHASTRE, J. C.; LEVASSEUR, J. P.; GIBERT, C.; PAIRE, A.; CABROL, C. - Prosthetic valve endocarditis with ring abscesses. J. Thorac. Cardiovasc. Surg., 105: 1106-1113, 1993.

5 JUBAIR, K. A.; FAGIH, M. R. A.; ASHMEG, A.; BELHAJ, M.; SAURGER, W. - Cardiac operations during active endocarditis. J. Thorac. Cardiovasc. Surg., 104: 487-490, 1992.

6 KEYE, D. - Endocardite infecciosa. In: WILSON, I. D.; BRAUNWALD, E.; ISSELBACHAVE, K. L. (eds.) Harrison: Medicina interna. Rio de Janeiro, 12. ed., 1992. p. $562-567$.

7 KIRKLIN, J. W. \& BARRAT-BOYES, B G. - Cardiac Surgery. 2. ed. New York, Hurchill Livingstone, 1993. p. 547.

8 KORZENOWSKI, O. M. \& KAYE, D. - Infective endocarditis In: BRAUNWALD, E. (ed.) Heart disease. 4. ed. Philadelphia, W. B. Saunders, 1992. p. $1078-1105$. em portadores de endocardite infecciosa. São Paulo, 1987. [Tese. Mestrado. Faculdade de Medicina da Universidade de São Paulo].

10 MANSUR, A. J. - Endocardite infecciosa. In: BARRETO A. C. P. \& SOUZA, A. G. M. R. (eds.) Cardiologia: atualizaçāo e reciclagem. Rio de Janeiro, SOCESP, 1994. p. $455-465$.

11 MANSUR, A. J.; GRIMBERG, M.; GALLUCCI, S. D. D. - Endocardite infecciosa: análise de 300 episódios. Arq. Bras. Cardiol., 54: 13-21, 1990.

12 MANSUR, H. \& JOHNSON, W.D. - Prosthetic valve endocarditis. J. Thorac. Cardiovasc. Surg., 80:3137,1980 .

13 McGIFFIN, D. C.; GALBRAITH, A. J.; McLACHLAN, G. J.; STOWER, R. E.; WONG, M. L.; STAFFORD, E. G.; GARDNER, M. A. H.; POHLNER, P. G.; O'BREIN, M. F. - Aortic valve infection: risk factors for death and recurrent endocarditis after aortic valve replacement. J. Thorac. Cardiovasc. Surg., 104: 511-520, 1992.

14 ROCCHICCIOLI, C.; CHASTRE, J. LECOMPTE, Y.; GANDJBAKHCH, I.; GIBERT, C. - Prosthetic valve endocarditis: the case for prompt surgical management. J. Thorac. Cardiovasc. Surg., 92: 784$789,1986$.

15 SETT, S. S.; HUDON, M. P. J.; JAMIESON, W. R. E.; CHOW, A. W. - Prosthetic valve endocarditis: experience with porcine bioprosthesis. J. Thorac. Cardiovasc. Surg., 105: 428-434, 1993.

16 SKEHAN, J. D.; MURRAY, M.; MILLS, P. G. - Infective endocarditis: incidence and mortality in the North East Thames Region. Br. Heart J.., 59: 62-68, 1988.

17 WATANAB, G. O.; HAVERICH, A.; SPEIER, R.; DRESLER, C.; BORST, H. G. - Surgical treatment of active infective endocarditis with paravalvular involvement. J. Thorac. Cardiovasc. Surg., 107: 171-177, 1994. 